\title{
Quasistatic elastic-plastic loading and unloading of rods with coulomb dry friction
}

\author{
Nigora Mamatova* \\ National University of Uzbekistan, Tashkent, Uzbekistan
}

\begin{abstract}
Loading of elastic-plastic rods with account on Prandtl's scheme is considered in the paper, and nonlinear interactions are taken into account under the law of Coulomb friction. Exact analytical solutions of quasi-stationary problems of interaction of elastic-plastic rod and undeformable media surrounding it are given.
\end{abstract}

\section{Introduction}

In works [1-11], the problem of quasistatic loading of structural elements in the form of an elastic rod interacting with its environment according to the Coulomb dry friction law was formulated and solved. It is known that in the problems of longitudinal vibrations of rods interacting with soil media, attention is drawn to the fact that in certain situations, it is possible to neglect the inertial terms in the equations of motion (works [12, 16, 20, 21], etc.) in comparison with the arising shear stresses on the surfaces of interaction between the structure and the environment. In this case, quasistatic formulations of nonlinear problems arise.

The paper considers the loading of an elastic-plastic rod according to the Prandtl scheme, and nonlinear interactions are taken into account according to the Coulomb dry friction law. Exact analytical solutions of quasi-stationary problems on the interaction of an elastic-plastic rod and the non-deformable soil environment surrounding it are given. The loading of the rod particles along two loaded sections of the Prandtl diagram and the unloading of the elastoplastic rod is taken into account. Rod structures are assumed to be long enough, which are found in deep drilling structures but loaded in the longitudinal direction.

\section{Methods}

\subsection{Statement of the problem of dynamic loading of a bar structure and a method for its solution}

Let a semi-infinite elastoplastic rod, which is in the initial state in contact with its surface with a non-deformable medium, be loaded from the end section $\mathrm{x}=0$ (the $\mathrm{x}$-axis is directed

\footnotetext{
*Corresponding author: niginahon@inbox.ru
} 
along the rod axis and $x>0$ ). Compressive loading at $x=0$ is reduced smoothly from zero, and it is set according to the following scheme (Figure 1).

Figure 2, the diagram of elastic-plastic loading and unloading of the rod particles according to the Prandtl scheme is shown. The loading given at $\mathrm{x}=0$ can be determined by the following analytical representation:

$$
\begin{gathered}
-\sigma_{0}(t)=\left\{\begin{array}{l}
-\sigma_{0}(t), \text { if a } 0 \leq t \leq t_{1} \\
-\tilde{\sigma}_{0}(t), \text { if a } t_{1} \leq t \leq t_{2} \\
-\sigma_{1}(t), \text { if a } t \geq t_{2}
\end{array}\right. \\
-\sigma_{0}\left(t_{1}\right)=-\tilde{\sigma}_{0}\left(t_{1}\right)=-\sigma_{s},-\tilde{\sigma}_{0}\left(t_{2}\right)=-\sigma_{1 m}\left(t_{2}\right) .
\end{gathered}
$$

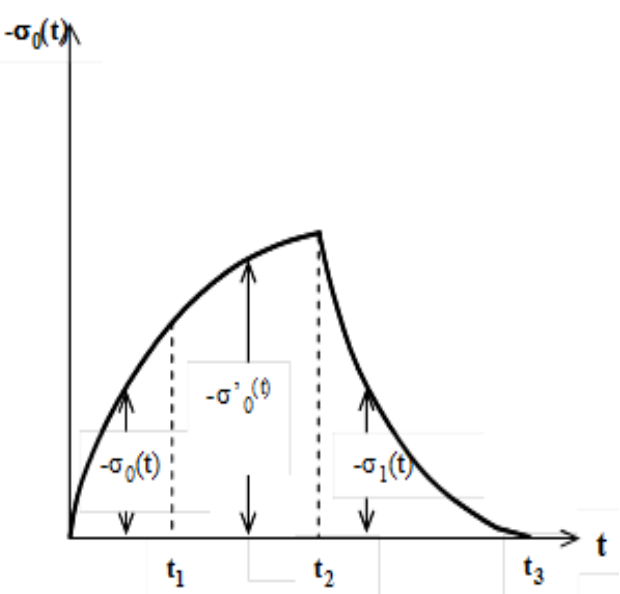

Fig. 1. Compressive loading at $x=0$

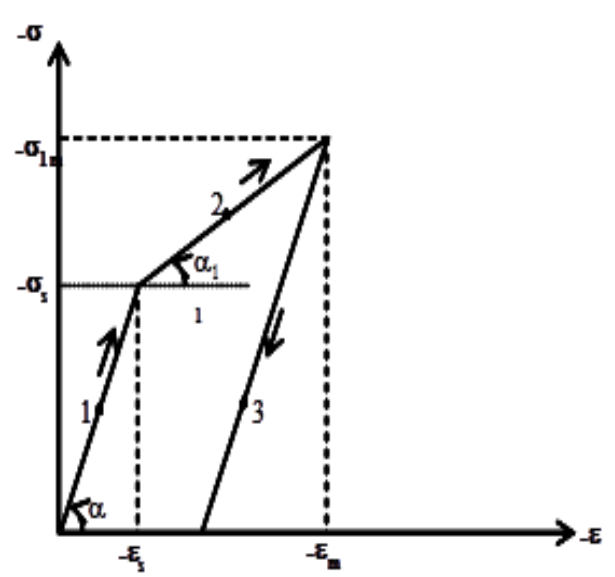

Fig. 2. The diagram of elastic-plastic loading and unloading of the rod particles according to the Prandtl scheme is shown

The values refer to the limit values for elastic strains and stresses. In accordance with the diagram (Fig. 2) for points, we have:

1. $\sigma=E \varepsilon$

2. $\sigma=E_{1} \varepsilon+\left(\sigma_{S}-E_{1} \cdot \varepsilon_{S}\right)$

3. $\sigma=E \varepsilon-\left(E-E_{1}\right)\left(\varepsilon_{m}-\varepsilon_{S}\right)$

where $\sigma_{1 m}$ and $\varepsilon_{m}$ refer to the beginning of unloading.

If there is an active compressive loading of the end section of the rod, then the resistance of the soil medium develops along the length of the rod. Neglecting the inertial terms for the loading sections (points 1 and 2 in Fig. 2), we have the following equation: $\frac{d \sigma}{d x}=\tau$. For the unloading sections, we have the equation $\frac{d \sigma}{d x}=-\tau$, where $\tau$ are the increments of shear stresses on the contact surface.

Note that the time $t$ appears in this work as a parameter and derivatives concerning it; in the basic equations of motion, they are equal to zero. 
Obviously, smooth loading and then unloading of the rod particles at $x=0$ leads to the need to use different equilibrium equations in the loading and unloading zones. Suppose the loading and unloading process is considered only within the limits of elasticity, then in the areas of loading and unloading of a semi-infinite bar. In that case, the boundaries of these areas, which change with time, must be determined. Solutions to problems in these areas must be built under satisfying conditions for the continuity of displacements and stresses at the boundaries. Suppose there is a monotonic loading of the section $\mathrm{x}=0$ according to Fig. 1 , then with the passage beyond the elastic limit. In that case, two loading zones are formed: the area of plastic and elastic deformations of the bar. Solutions in areas with movable boundaries, boundaries, and conditions on them must be determined in the process of solving problems. The analytical construction of problems becomes more complicated if the particles of the rod are unloaded from the states corresponding to points 2 (Fig. 2).

In the area for points 1 in Figure 1, when there is a loading process, which does not exceed $\sigma_{s}$ at the end section, we have:

$$
\begin{gathered}
\left\{\begin{array}{c}
\sigma=-\sigma_{0}+\tau x \\
u(x, t)=\frac{\sigma_{0}(t)}{E}\left(l_{s 1}-x\right)-\frac{\tau}{2 E}\left(l_{s 1}^{2}-x^{2}\right) \\
l_{s 1}=\frac{\sigma_{0}(t)}{\tau}, 0 \leq x \leq l_{s 1} . \\
\left\{\begin{array}{l}
\sigma=0 \\
u=0
\end{array}, x \geq l_{s 1}\right.
\end{array}\right.
\end{gathered}
$$

These formulas were also obtained in [2-4].

In the region of loading beyond the elastic limit, when $|\sigma| \geq \sigma_{S}$ we have

$$
\sigma=E_{1} \varepsilon+\left(\sigma_{S}-E_{1} \varepsilon_{S}\right) \text { and } \frac{d \sigma}{d x}=\tau
$$

We integrate the second equation (2) for a fixed time $\mathrm{t}$ from zero to $\mathrm{x}$ :

$$
\sigma(x, t)=\sigma(0, t)+\tau x
$$

Here $t=t 1+t^{\prime}\left(t^{\prime}>0\right)$ and for times $t>t 1$ we have: $\sigma(x, t)=-\widetilde{\sigma}_{0}(t)+\tau x$

At $\quad t=t_{1}: \quad \sigma\left(x, t_{1}\right)=-\widetilde{\sigma}_{0}\left(t_{1}\right)+\tau x=-\sigma_{s}+\tau x$

At $\quad t=t_{2}: \quad \sigma\left(x, t_{2}\right)=-\widetilde{\sigma}_{0}\left(t_{2}\right)+\tau x=-\sigma_{m}\left(t_{2}\right)+\tau x$

Based on the first relation (2), we have:

$$
E_{1} \frac{\partial u}{\partial x}+\left(\sigma_{s}-E_{1} \varepsilon_{s}\right)=-\widetilde{\sigma}_{0}(t)+\tau x
$$

From here, we get: 


$$
u(x, t)-u(0, t)=\frac{\tau x^{2}}{2 E_{1}}-\frac{\widetilde{\sigma}_{0}(t)}{E_{1}} x+\varepsilon_{s} x-\frac{\sigma_{s}}{E_{l}} x
$$

Solutions (5) are valid on the interval $0<\mathrm{x}<\mathrm{ls} 2(\mathrm{t})$, where the boundary relations for elastically and plastically deformable regions can be used to determine ls2 (t):

$$
-\sigma_{S}=-\tilde{\sigma}_{0}(t)+\tau \cdot l_{s 2}
$$

Hence we have: $l_{s 2}=\frac{\tilde{\sigma}_{0}(t)-\sigma_{s}}{\tau}$

In the region $1 \mathrm{~s} 2(\mathrm{t})<\mathrm{x}<\mathrm{lsl}(\mathrm{t})$, where the rod is deformed within the elasticity, we again have:

$$
\frac{d \sigma}{d x}=\tau
$$

Integrating it from ls2 (t) to $\mathrm{x}$, we have:

$$
\sigma=-\sigma_{S}+\tau\left(x-l_{s 2}\right)
$$

where is the border $l_{s 1}=\sigma_{s} / \tau+l_{s 2}(t)$.

Considering that here $\sigma=E \frac{\partial u}{\partial x}$ and integrating, we find:

$$
u(x, t)=u\left(l_{s 2}, t\right)-\frac{\sigma_{s}}{E}\left(x-l_{s 2}\right)+\frac{\tau}{2 E}\left(x-l_{s 2}\right)^{2}, \text { где } l_{s 2} \leq x \leq l_{s 1}
$$

Relation (7) makes it possible to determine $u(l s 2, t)$ from the condition $u(l s 1, t)=0$. Given this circumstance, we get:

$$
u(x, t)=\frac{\sigma_{s}}{E}\left(l_{s 1}-x\right)+\frac{\tau}{2 E}\left[x^{2}-l_{s 1}^{2}+2 \cdot l_{s 2}\left(l_{s 1}-x\right)\right], \text { where } l_{s 2} \leq x \leq l_{s 1}
$$

If we substitute the values of the above expressions for $1 \mathrm{~s} 1$ and $1 \mathrm{~s} 2$, then after some calculations, we get:

$$
u(x, t)=\frac{1}{2 E \tau}\left[\tilde{\sigma}_{0}(t)-\tau x\right]^{2}, \text { where } l_{s 2} \leq x \leq l_{s 1}
$$

from (5) and (9), we get: 


$$
\begin{gathered}
u\left(l_{s 2}, t\right)=u(0, t)-\frac{\sigma_{s}}{E_{1}} l_{s 2}+\varepsilon_{s} \cdot l_{s 2}-\frac{\tilde{\sigma}_{0}(t)}{E_{1}} \cdot l_{s 2}+\frac{\tau}{2 E_{1}} \cdot l_{s 2}{ }^{2} \\
u\left(l_{s 2}, t\right)=\frac{1}{2 E \tau}\left[\tilde{\sigma}_{0}(t)-\tau \cdot l_{s 2}\right]^{2}
\end{gathered}
$$

equating the right-hand sides (10) and (11) we get:

$$
u(0, t)=\frac{1}{2 E \tau}\left[\tilde{\sigma}_{0}(t)-\tau \cdot l_{s 2}\right]^{2}+\frac{\sigma_{s}}{E_{1}} l_{s 2}-\varepsilon_{s} \cdot l_{s 2}+\frac{\tilde{\sigma}_{0}(t)}{E_{1}} \cdot l_{s 2}-\frac{\tau}{2 E_{1}} \cdot l_{s 2}{ }^{2}
$$

we substitute (12) into (5), and after substituting the expressions for 1s1 and ls2 we get:

$$
u(x, t)=\frac{\tau}{2 E_{1}} x^{2}+\frac{\sigma_{S}^{2}}{2 E \tau}-\left(\frac{\tilde{\sigma}_{0}(t)+\sigma_{s}}{E_{1}}-\varepsilon_{S}\right) x-\frac{\tilde{\sigma}_{0}-\sigma_{s}}{\tau} \cdot \varepsilon_{S}+\frac{3 \tilde{\sigma}_{0}^{2}-\sigma_{s}^{2}-2 \tilde{\sigma}_{0} \sigma_{s}}{2 E_{1} \tau}
$$

Thus, under monotonic loading of a semi-infinite bar in a region plastically deformed with a movable boundary, stresses and displacements are calculated by the formulas:

$$
\left\{\begin{array}{c}
\sigma(x, t)=-\widetilde{\sigma}_{o}(t)+\tau x \\
u(x, t)=\frac{\tau}{2 E_{l}} x^{2}+\frac{\sigma_{s}^{2}}{2 E \tau}-\left(\frac{\widetilde{\sigma}_{0}(t)+\sigma_{s}}{E_{l}}-\varepsilon_{s}\right) x-\frac{\widetilde{\sigma}_{0}-\sigma_{s}}{\tau} \cdot \mathcal{E}_{s}+\frac{3 \widetilde{\sigma}_{0}^{2}-\sigma_{s}^{2}-2 \widetilde{\sigma}_{0} \sigma_{s}}{2 E_{l} \tau}
\end{array}\right.
$$

where $0 \leq x \leq l_{s 2}$.

We add here the solutions obtained above in the elastically deformable region:

$$
\left\{\begin{array}{c}
\sigma=-\sigma_{s}+\tau\left(x-l_{s 2}\right) \\
u(x, t)=\frac{1}{2 E \tau}\left[\tilde{\sigma}_{0}(t)-\tau x\right]^{2}, \text { where } l_{s 2} \leq x \leq l_{s 1}
\end{array}\right.
$$

It is not difficult to make sure that stresses and displacements are continuous functions at the moving boundary between the regions $l_{s 2}=\frac{\tilde{\sigma}_{0}(t)-\sigma_{s}}{\tau}$.

In the unloading area

$$
\begin{gathered}
\frac{d \sigma}{d x}=-\tau \\
\sigma(x, t)=-\sigma_{1}(t)-\tau x, l_{s 3}(t)=\frac{\sigma_{1 m}\left(t_{2}\right)-\sigma_{1}(t)}{2 \tau}
\end{gathered}
$$

At the moment $t=t_{2}$ from $0 \leq x \leq l_{s 2}$, we have: 


$$
\frac{\partial u}{\partial x}=-\frac{\tilde{\sigma}_{0}\left(t_{2}\right)}{E_{1}}+\frac{\tau x}{E_{1}}+\frac{\sigma_{s}}{E_{1}}-\varepsilon_{S}=-\frac{\sigma_{1 m}\left(t_{2}\right)}{E_{1}}+\frac{\tau x}{E_{1}}+\frac{\sigma_{s}}{E_{1}}-\varepsilon_{S}
$$

When unloading, we have:

$$
\sigma=E \varepsilon-\left(E-E_{1}\right)\left(\varepsilon_{m}(x)-\varepsilon_{s}\right)
$$

From (16), we have:

$$
-\sigma_{1}(t)-\tau x=E \frac{\partial u(x, t)}{\partial x}-\left(E-E_{1}\right)\left(\varepsilon_{m}(x)-\varepsilon_{s}\right)
$$

The boundary $x=l_{s 3}(t)$ is the leading front of unloading, and in the $0 \leq x \leq l_{s 3}$ sections for the beginning of unloading, the deformation is equal $\varepsilon_{m}(x)$. For each section $\mathrm{x}^{*}$, where $0 \leq x^{*} \leq l_{s 3}$, you can determine the start time of unloading from the equation:

$$
\begin{aligned}
x^{*}=l_{s 3}, \text { that is, from } x^{*} & =\frac{\sigma_{1 m}\left(t_{2}\right)-\sigma_{1}(t)}{2 \tau} . \\
\sigma_{1}(t) & =\sigma_{1 m}\left(t_{2}\right)-2 \tau \cdot x^{*}=\psi\left(t_{2}, x^{*}\right)
\end{aligned}
$$

we have: $t^{*}=\psi^{*}\left(t_{2}, x^{*}\right)$, where $\psi^{*}$ is the inverse function $\psi\left(t_{2}, x^{*}\right)$ of the function at a constant value of $t_{2}$. Then

$$
\left.\sigma(x, t)\right|_{\substack{t=t^{*} \\ x=x^{*}}}=-\sigma_{1}\left(t^{*}\right)-\tau \cdot x^{*}=-\sigma_{m}\left(x^{*}\right)
$$

Then from (17), we have

$$
\varepsilon_{m}\left(x^{*}\right)=-\frac{\sigma_{m}\left(x^{*}\right)}{E_{1}}-\frac{E-E_{1}}{E_{1}} \cdot \varepsilon_{S}
$$

Thus, for each fixed $x^{*}$, we find $\sigma_{m}\left(x^{*}\right)$ и $\varepsilon_{m}\left(x^{*}\right)$.

From (18), if $\varepsilon_{m}(x)$ there is a known function of $\mathrm{x}$, then

$$
E \frac{\partial u(x, t)}{\partial x}=\left(E-E_{1}\right)\left(\varepsilon_{m}(x)-\varepsilon_{S}\right)-\sigma_{1}(t)-\tau x
$$




$$
u(x, t)-u(0, t)=-\frac{\sigma_{1}(t)}{E} x-\frac{\tau}{E} x^{2}+\int_{0}^{x} \frac{\left(E-E_{1}\right)}{E} \varepsilon_{m}(x) d x-\frac{\left(E-E_{1}\right)}{E} \varepsilon_{S} x
$$

At the boundary during $x=x^{*}$ displacement should be continuous, equating (20) and (14), we determine $u(0, t)$ and substituting it into (20), we find $u(x, t)$ when $x \in\left[0, l_{s 3}\right]$.

$$
\begin{aligned}
& u(x, t)=\frac{\tau}{2 E_{1}} l_{s 2}^{2}-\left(\frac{\tilde{\sigma}_{0}\left(t_{2}\right)-\sigma_{s}}{E_{1}}+\varepsilon_{s}\right) l_{s 2}+\frac{\tilde{\sigma}_{0}\left(t_{2}\right)-\sigma_{s}}{\tau} \varepsilon_{s} \\
& +\frac{\left(\tilde{\sigma}_{0}\left(t_{2}\right)-\sigma_{s}\right)^{2}}{2 E_{1} \tau}+\frac{\sigma_{s}^{2}}{2 E \tau}+\frac{\tau}{2 E}\left(l_{s 2}{ }^{2}-x^{2}\right)+\left(l_{s 2}-x\right) . \\
& \left(\frac{\sigma_{1}(t)}{E}+\frac{\left(E-E_{1}\right)}{E} \varepsilon_{S}\right)+\frac{\left(E-E_{1}\right)}{E}\left\{\left(\frac{1}{E_{1}} \sigma\left(x^{*}, t^{*}\right) x-\frac{\left(E-E_{1}\right)}{E} \varepsilon_{S} x\right)-\Delta x_{1} \sum_{i=1}^{n} \varepsilon_{m}\left(x_{i}\right)\right\}
\end{aligned}
$$

\section{Results and Discussion}

\subsection{Numerical example of solving the problem of loading and unloading a rod with external dry friction.}

Let a $\sigma_{0}(t)$ stress be applied to the end $x=0$ of the elastoplastic rod, which increases to the value $a_{0}$, and starting from the moment of time $t_{2}$, the unloading of the rod particles occurs.

The end of the bar $x=0$ is loaded according to a given law

$$
\sigma_{0}(t)=\left\{\begin{array}{cc}
-a_{0} \sin \omega t, & 0 \leq t \leq t_{1} \\
-a_{0} \sin \omega t, & t_{1} \leq t \leq t_{2} \\
-\sigma_{1 m}\left(t-t_{3}\right) /\left(t_{2}-t_{3}\right), t_{2} \leq t \leq t_{3}
\end{array}\right.
$$

$a_{0}=135$ MПа; $\omega=1$ рад / сек.

$t_{1}=0.73$ сек, $t_{2}=1.336$ сек, $t_{3}=2$ сек.

Let us take the following initial data for deformable media: material its low carbon steel (HRB 77) with parameters $E=2.1 * 10^{5} \mathrm{MPa}, \quad \gamma=7800 \mathrm{KG} / \mathrm{m} 3, \quad \sigma_{s}=90 \mathrm{MPa}, \quad \varepsilon_{S}=0.00043$, $\sigma_{1 m}=132 \mathrm{MPa}, E_{1}=0.1 \cdot \mathrm{E} \mathrm{MPa}$ is linear hardening module. At $v=0.3, \tau^{+}=11.428 \mathrm{MPa} / \mathrm{m}$ is the shear stress at the interface between the rod and the medium. 


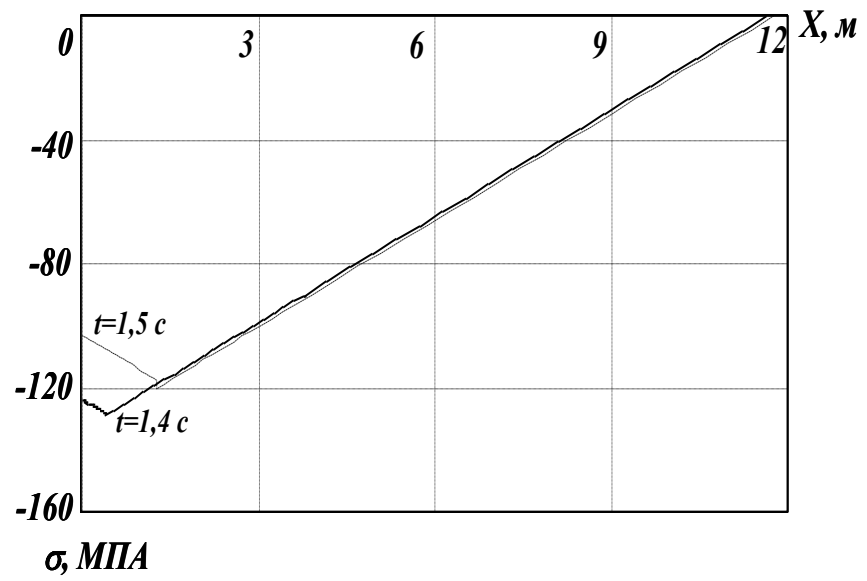

Fig. 3. Distribution of longitudinal stresses along the length of the bar for fixed points in time.

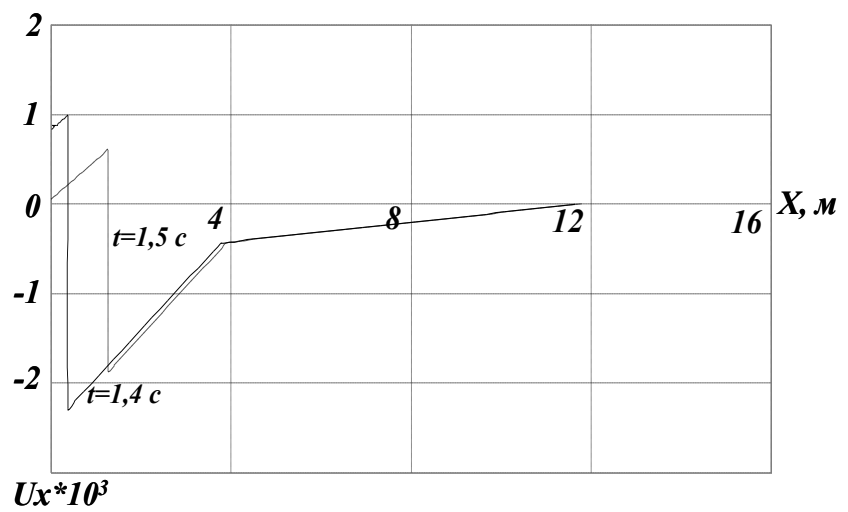

Fig. 4. Distribution of longitudinal deformations of the bar sections along the length.

Figure 3-4 show the graphs of changes in longitudinal stresses and deformations in unloading zones, elastic-plastic and elastic zones for fixed points in time. Area $0<x<1.26$ - corresponds to the unloading zone, $1.26<x<3.77$ - to the elasticplastic zone, $3.77<x<11.78$ - to the elastic zone, for different moments of time.

Figure 4 shows that in the unloading region, the deformation increases in absolute value, i.e., the structure is stretched and then abruptly passes into the plastic zone, decreasing along the length of the rod.

In the unloading zone, the stress increases until the time $\mathrm{t} 3$, then gradually decreases along the length of the rod.

\section{Conclusions}

1. The problem of quasistatic loading and unloading of long bar structures in an elastic medium and interacting with it according to the Coulomb dry friction law has been solved. Analytical solutions are given for quasi-stationary problems for an elastic-plastic rod and its surrounding soil medium. 
2. The boundaries of movable elastic, elastoplastically loading surfaces of the rod and the area where the particles of the rod are unloaded are determined.

3. Exact analytical formulas developed parameters in deformable areas of impact, which can be used in quasistatic formulations of the structure and environment.

\section{References}

1. Sultanov K.S., Vatin N.I. Wave Theory of Seismic Resistance of Underground Pipelines. Appl. Sci. 11, 1797. (2021)

2. Nikitin L.V. Dynamics of elastic rods with external dry friction, Uspekhi mekhaniki. 11. (4). (1988)

3. Nikitin L.V. Wave propagation in an elastic rod in the presence of dry friction, Ing. magazine MTT.3. (1963)

4. Nikitin L.V. Statics and dynamics of rigid bodies with external friction. Moscow Lyceum, (1998)

5. Nikitin L.V., Tyurekhodzhaev A.N. Impact of a shock wave in the ground on an underground pipeline, Izv. USSR Academy of Sciences, MTT. (1). (1987)

6. Rashidov T.R. Dynamic theory of seismic resistance of complex systems of underground structures6 Tashkent, (1973)

7. Nikitin L.V., Tyurekhodzhaev A.N. Behavior under load of an elastic rod buried in the ground, Problems of rock mechanics. Almaata: Science, (1966)

8. Nikitin L.V., Tyurekhodzhaev A.N. Behavior of the pipeline under the action of a shock wave in the soil, Mater. int. scientific. conf. Friction, Wear and Lubricants. Tashkent, (2). (1985)

9. Nikitin L.V., Tyurekhodzhaev A.N. Underground pipeline under the influence of a shock wave in the ground, Izv. Academy of Sciences of the USSR. Ser. those. sciences. 5. (1986)

10. Cornelius C.S., Kubitza W.K. Experimental investigation of longitudinal wave propagation in an elastic road with Coulomb friction, Exp. Mech. 10. (4), (1970)

11. Mogilevsky R.I., Ormonbekov T.O., Nikitin L.V. Dynamics of rods with interfacial dry friction, J. Mech. Behav. Mater. 5(1). (1993)

12. Nikitin L.V., Fischer F.D., Oberaigner E.R., Rammestorfer F.G., Sietzberger M., Mogilevsky R.I. On the frictional behavior of the thermally loaded resting on a plane, Int. J. Mech. Sci. 38. (11), (1996)

13. Nikitin L.V., Tyurekhodgaev A.N. Wave propagation and vibration of elastic rods with interfacial frictional slip, (12). (1990)

14. Shah V.N., Gilmore C.B. Seismic analysis of structures with Coulomb friction, Trans. ASME. J. Pressure Vessel Technol. 105 (2), (1983)

15. Martin h. Muser, Michael urbakh, Mark o. Robbins. Statistical mechanics of static and low-velocity kinetic friction. Advances in Chemical Physics, 126, (2003)

16. Petrashen G.I. Foundations of the mathematical theory of propagation of elastic waves, Problems of the dynamic theory of seismic wave propagation, Issue XVIII. Nauka, p. 248, (1978)

17. The victory of B.E. Numerical methods in the theory of elasticity and plasticity, M. Moscow State University, p. 344, (1981)

18. Lieutenant V.B. Methods of the dynamic theory of elasticity, Moscow, Nauka, p. 328. (1986)

19. Rabotnov Yu.N. Mechanics of a deformable solid, Moskva, Nauka, p.712. (1988)

20. Rakhmatulin Kh.A. Gas and wave dynamics. M, Mosk. University, (1983)

21. Rakhmatulin Kh.A., Durability under intense short-term loads: Fizmatgiz, (1961) 